\title{
In-bore Electric and Magnetic Field Environment
}

\author{
Alexander E. Zielinski and Calvin D. Le \\ U.S. Army Research Laboratory, Aberdeen Proving Ground, Maryland 21005-5066 \\ John A. Bennett \\ U.S. Army ARDEC, Picatinny Arsenal, New Jersey, 07801-5000
}

\begin{abstract}
A jointly sponsored ARDEC-ARL effort is under way to investigate and evaluate the electromagnetic (EM) field environment to which launch package electronics will be exposed during EM launch. In addition, exposure trials were performed on electronic circuit components, with diagnostic tests conducted before and after exposure to establish that the hardware is working properly or to ascertain the existence/nature of an induced malfunction.

Tests for the in-bore environment are conducted for a stationary armature in a short section of a $50-\mathrm{mm}$ railgun. Two contact states are studied: solid and arcing (i.e., transitioned). The magnetic field ahead of the armature is measured as a function of axial position. The measurements are made in the same location where the circuit components are located. A $500-\mathrm{MHz}$ high voltage probe is used to measure the voltage at the muzzle of the railgun, and the electric field is measured outside the gun bore. Based on these measurements, the in-bore electric field content will be inferred. Both the electric and magnetic fields are evaluated, based on their spectral content and electronic circuit component functionality.
\end{abstract}

Index Terms-Electric field, Magnetic field, Measurements, Spectra

\section{Introduction}

Electromagnetic armaments are part of a comprehensive emerging weapon system that is important to the armed services. Included in the category are the railgun, induction plate launcher, electrothermal (ET) gun, and the ellectrothermal chemical (ETC) gun. The electric and magnetic (EM) field environments generated by these devices and their associated pulsed power systems have not been experienced before in fielded military applications. These fields may be of concern for the traditional electromagnetic interference (EMI) and electromagnetic compatibility (EMC) effects on collateral equipment, but may also affect biological systems as well as the functionality of precision guided munitions (PGM) while residing in bore. This paper addresses preliminary measurements relative to in-bore fields and their effects.

Also of concern is the spectral content of the in-bore environment. The railgun generates EM fields whose domi- nant energy spectrum lies between tens of hertz $(\mathrm{Hz})$ and tens of $\mathrm{kHz}$. However, for certain cases of operation such as transitioned armature contacts, higher frequency components may be excited. These, albeit much diminished in magnitude, may in turn readily couple to PGM subcomponents. Also, in PGMs, the coupling mechanism may be deliberate (e.g., through a transmitting or receiving antenna). This then makes shielding the PGM from the in-bore environment impractical.

Although all the aforementioned launcher systems are in the prototype stages of development the in-bore environment should be addressed. It is much more economical to identify and correct potential issues during the design phase than in the development phase. The EMC and EMI issues can be defined from theoretical predictions and experimental observations of laboratory models. With only a few exceptions, the environmental results will scale to the actual system dimensions. This type of data is invaluable to the PGM designer as sensitive subcomponents can be more judiciously located relative to the field generation source.

The remainder of the paper is organized as follows. In Section II, an analytical expression is derived for the magnetic field (B-field). The electric field (E-field) is briefly considered. In Section III, the experiments conducted to ascertain the field data are presented. These results include the magnitude of the B-field, the spectral content, and the component exposure tests. Finally, the summary and preliminary conclusions are presented.

\section{Theoretical}

The essential features of the $50-\mathrm{mm}$ square-bore single turn railgun are illustrated in Fig. 1. The railgun is comprised of two copper rails, 0.63-m long and having a cross section of $60 \mathrm{~mm} \times 25 \mathrm{~mm}$. The bore insulators are fabricated from a fiberglass-epoxy laminate (G-11). Also shown in the photograph is the armature, armature in-bore restraint, and the electronics package.

A mathematical representation of the B-field for the magnetostatic system is developed from the familiar Biol and Savart's Law:

$$
\vec{B}(\vec{r}, t)=\frac{\mu_{o}}{4 \pi} \int \frac{\vec{J}\left(\vec{r}^{\prime}, t\right) \times\left(\vec{r}-\vec{r}^{\prime}\right)}{\left|\vec{r}-\vec{r}^{\prime}\right|^{3}} d v^{\prime}
$$

A. Zielinski, E-mail: zielinsk@arl.mil, Fax: 410-278-2460; C. Le E-mail: cle@arl.mil, J. Bennett, E-mail: jbennett@pica.army.mil 


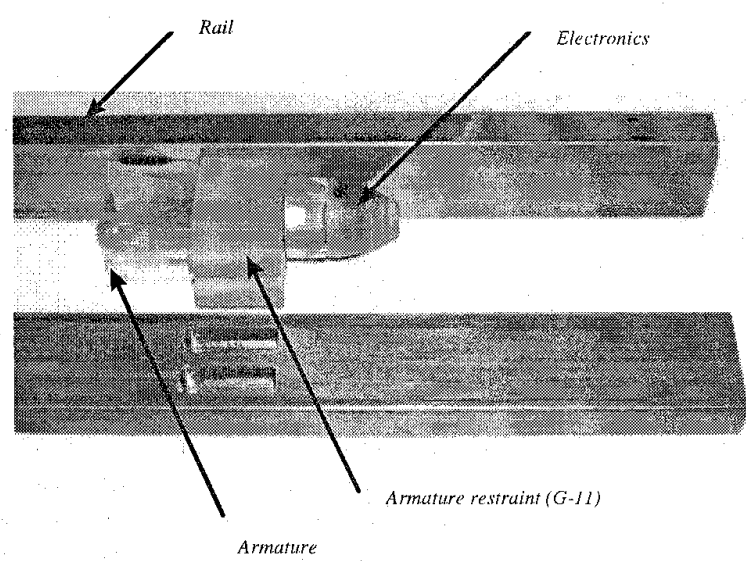

Fig. 1. Photograph of rails, armature, and electronics package.

From Fig. 1, the x-direction is taken along the centerline of the railgun, with the height of the rail in the $z$-direction and the $y$-axis directed from rail to rail. The origin of the system is at the center of the armature. The B-field is to be calculated for a sheet of total current, I, and constant linear current density, $I^{\prime}=I / a$, in which $a$ equals the height of the rails. The rails are separated by a distance $d$, and the rails are infinitely long in the negative $\mathrm{x}$-direction. Since it is the $\mathrm{z}$-component of the B-field along the centerline that is primarily of interest, simplification of the result is achieved by considering only that special case $(y=z=0)$. The expression for the azimuthal component of the in-bore B-field as a function of axial location, $B_{z}(\mathrm{x})$, is given by

$$
B_{z}=\frac{\mu_{o} I}{\pi a}\left\{\arctan \left(\frac{a}{d}\right)-\arctan \left(\frac{a R}{x d}\right)\right\}
$$

in which

$$
R=\sqrt{x^{2}+\frac{d^{2}}{4}+\frac{a^{2}}{4}}
$$

It is not possible to directly measure the in-bore E-field. Therefore, comparisons between theory and experiment, for model validation, are not possible. Furthermore, prior work has shown that the E-field environment outside the launcher is complicated by external conductors and structures [1]. Therefore, the magnitude of the in-bore E-field at the electronic package location is inferred from the measured exterior data and scaled using the analytical expression $[1]$,

$$
\begin{aligned}
E_{y}= & -\frac{\mu_{o} \dot{I}}{4 \pi a}\{a \ln (R+\hat{y})+\hat{y} \ln (2 R+a)-\hat{y} \ln (2 R-a) \\
& -a \ln (R+\bar{y})-\bar{y} \ln (2 R+a)+\bar{y} \ln (2 R-a)\}
\end{aligned}
$$

and $\hat{y}=+\frac{d}{2}$ and $\bar{y}=-\frac{d}{2}$.

\section{Experiments.}

Limited quantity, uncalibrated magnetic field data have been measured ahead of a moving armature and successfully transmitted to an on-board data recording system [2]. For the tests presented in this paper, the armature was stationary in the bore of the railgun. In order to accomplish this with minimal perturbation of the inbore environment, an insulated restraint was affixed to the rails. The restraint was fabricated from G-11 and is held in place using pockets milled into the bore surface of the rails.

The instrumentation used to measure the environment consists of time rate of change in magnetic field $(\mathrm{dB} / \mathrm{dt})$ probes and monopole antenna E-field probes. The $\mathrm{dB} / \mathrm{dt}$ signal was measured using a seven-turn coil wound on a nylon form. The dimensions and turns were chosen as a compromise between high frequency capability and sufficient signal voltage [3]. Three sensors were wound on a single form to obtain three measurements in one test as a function of spatial location. Each sensor was calibrated in a transverse electromagnetic wave cell (TEM). A plot of one of the probe's attenuation as a function of frequency is shown in Fig. 2. The probe response is nearly flat to $6 \mathrm{MHz}$ where a resonance exists. The probe's 3-db level is $9 \mathrm{MHz}$. For frequencies above $9 \mathrm{MHz}$, additional resonances are present. The probe was also calibrated with its major sensing area rotated $90^{\circ}$ from the incident magnetic field wave. For this calibration, the magnitude of the output signal was several orders of magnitude below the calibration signal.

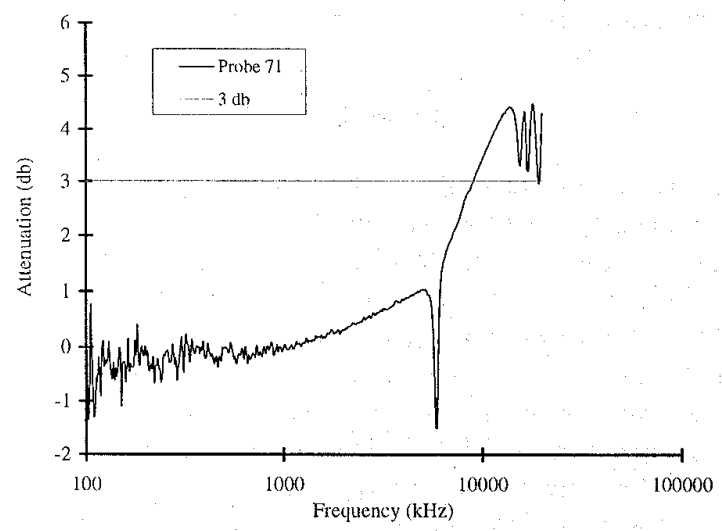

Fig. 2. Attenuation for the $d B / d t$ sensor

The E-field was measured using a monopole antenna and amplifier circuit. The probe has a linear response to $1 \mathrm{MHz}$. This instrumentation was used in a prior investigation and was found to be in reasonable agreement with a high-frequency, commercially available sensor [1]. The E-field probe was used to measure the rail-to-rail component $\left(E_{y}\right)$ outside the bore. This field component was found to be the largest.

The muzzle voltage, $V_{m}$, was measured using an isolated technique [1] with a frequency limit of $1 \mathrm{MHz}$ as well as 
with an active probe capable of measuring signals as great as $500 \mathrm{MHz}$. The high frequency data were acquired with a $200 \mathrm{MHz}$ digital storage oscilloscope. The AC power for the scope was provided by a gasoline powered generator so as to eliminate ground loops in the measurements.

\section{A. In-bore Fields}

The measurements of the fields are performed with the armature stationary. This is accomplished by placing an insulating restraint in the bore. By the interaction of the armature current and magnetic field produced by the rails, a force is generated in the armature. This force is reacted against the restraint and causes stress in the materials. Analytical expressions for the stress [4] and the material yield strength of the restraint were used to determine an acceptable axial length, $l_{r}$. The intention is to expose electronic components to the in-bore field. Therefore, the peak azimuthal component of the B-field $\left(B_{z}\right)$ is calculated from (2), at a distance, $\mathrm{x}=l_{r}+7 \mathrm{~mm}$ (i.e., down bore from the restraint). Additionally, the cross-sectional area of the armature was determined from action-integral considerations [5].

The results from this analysis are shown plotted in Fig. 3. The plot includes curves for restraint heights less than the rail height. From the figure there exists an optimum peak current in the armature to generate the largest $B_{z}$ down bore from the armature. For very small peak currents, $l_{r}$ is relatively small and the exposure location is quite close to the armature. However, since the current is small, so is $B_{z}$. On the other hand, even though a very large current generates quite a large $B_{z}$, the force generated by the armature is also large and $l_{r}$ increases accordingly to support the force. Hence, $B_{z}$ at the exposure location is significantly reduced.

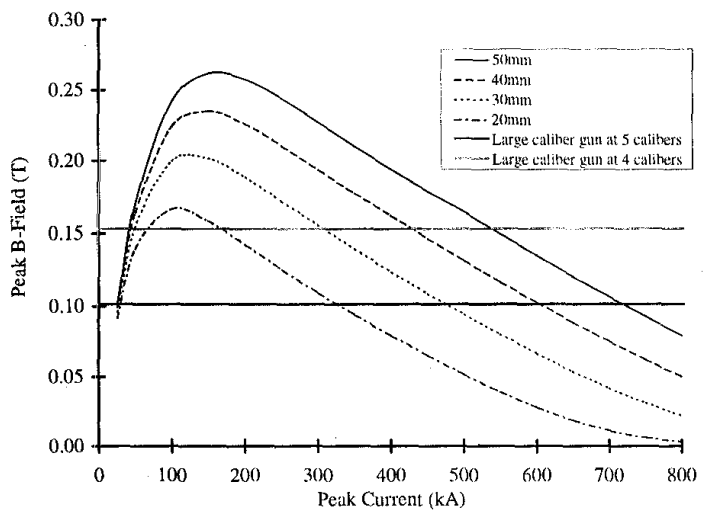

Fig. 3. Peak $B_{z}$ ahead of the in-bore armature restraint as a function of peak armature current. Plot includes calculations for various restraint heights and a large caliber hypervelocity railgun.

Also shown in the figure is the calculated $B_{z}$ for a large caliber hypervelocity railgun. The selected distances are representative of an electronic package located in a PGM. Clearly, there is no need to expose the electronics to an excessively large B-field (i.e., $>0.15 \mathrm{~T}$ ). Implementing the restraint in the $50-\mathrm{mm}$ bore further reduces the possible solutions to restraint heights of roughly $30 \mathrm{~mm}$. A peak current of $250 \mathrm{kA}$ and a restraint height of $31 \mathrm{~mm}$ were selected for the design. The axial length of the restraint is $48 \mathrm{~mm}$, and the exposure location is at $56 \mathrm{~mm}$. This current and dimensions allows for some flexibility in estimating the in-bore fields as well as improving the chances of generating a solid and transitioned contact from one armature design.

In Fig. 4, the measured and calculated $B_{z}$ is plotted as a function of $x$. The armature has an axial thickness at the front of $5 \mathrm{~mm}$. Therefore two calculations are presented since the equations are valid for a thin current sheet. The calculation using the rear of the armature as the reference is in better agreement with the data.

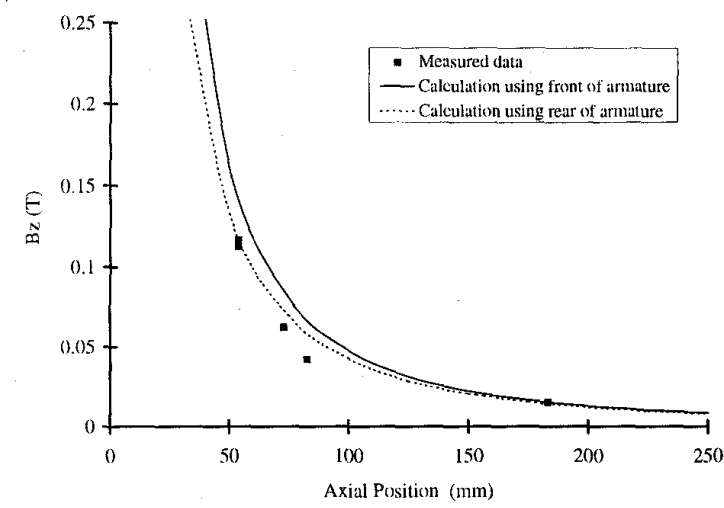

Fig. 4. Predicted and measured peak $B_{z}(\mathrm{x})$.

The probes were also used to measure $B_{z}$ behind the armature. In this case, the launcher dimensions are used with the measured peak current $(233 \mathrm{kA})$ and $B_{z}(1.9 \mathrm{~T})$ to obtain an inductance gradient of $0.41 \mu \mathrm{H} / \mathrm{m}$. This is in agreement with prior results [6].

The acquisition of the E-field data are described elsewhere [1]. The E-field has been found to be complicated by external conductors. For the purpose of establishing the in-bore E-field environment the total measured E-field and the contribution solely attributable to the armature and rail system are considered. In Fig. 5, the measured $E_{y}$ outside the launcher is plotted as a function of downrange location. The $\mathrm{E}$-field data were scaled to the in-bore E-field ahead of the armature. The resultant scaling constant and the analytical expression for the in-bore E-field (4) were then used to determine $E_{y}$ at $100 \mathrm{~mm}$ in front of the armature. These calculations are also indicated in the figure. The two values at $\mathrm{x}=100 \mathrm{~mm}$ are $3.3 \mathrm{kV} / \mathrm{m}$ for scaling the total field and $0.6 \mathrm{kV} / \mathrm{m}$ for scaling only the armature contribution. 


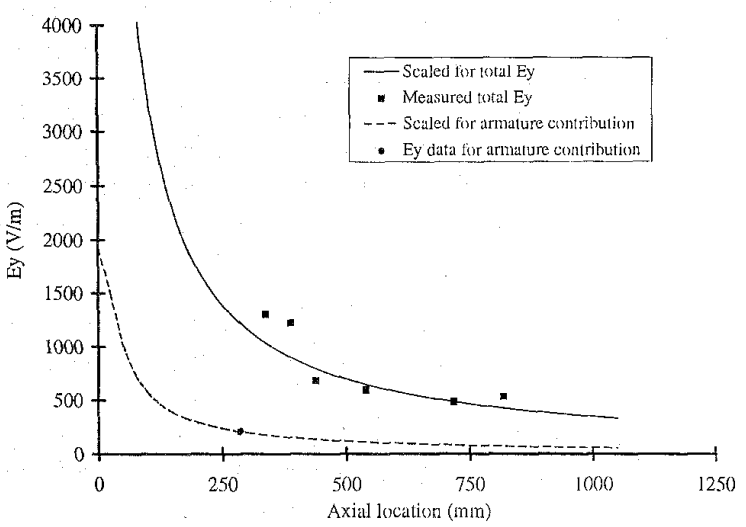

Fig. 5. Peak total $E_{y}(x)$ and armature contribution. (Data is from Reference 1).

\section{B. Spectral Content of Fields}

Measurements were made of the exterior E-field environment up to $1 \mathrm{MHz}$, the B-field environment up to $10 \mathrm{MHz}$, and the muzzle voltage up to $50 \mathrm{MHz}$. In all cases the spectrum was assessed by using the fast fourier transform (FFT) of the measured data.

The breech assembly was shorted and the voltage across the short was recorded at $20 \mathrm{MHz}$ using the $500-\mathrm{MHz}$ probe. A plot of the data as a function of time for the first $25 \mu \mathrm{s}$ is shown in Fig. 6. The initial transient caused by the switching of the ignitrons produces a noisy environment. In fact, the FFT of this data revealed two narrow-band peaks in the spectrum centered at $2.5 \mathrm{MHz}$ and $5 \mathrm{MHz}$. The $2.5 \mathrm{MHz}$ signal peak is also evident in another test using the in-bore $\mathrm{dB} / \mathrm{dt}$ probe recorded at $5 \mathrm{MHz}$.

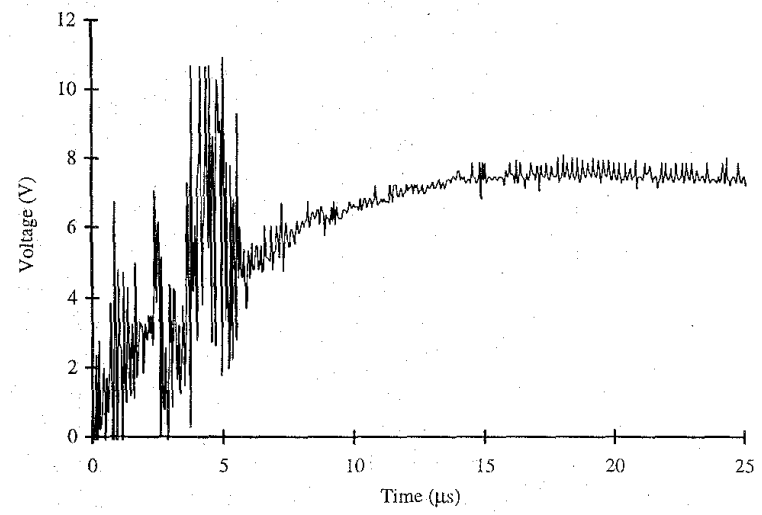

Fig. 6. Voltage measured across the shorted breech $(t \leq 25 \mu \mathrm{s})$.

The effect of contact transition on frequency content of the environment was evaluated. Data from the downrange E-field, in-bore $\mathrm{dB} / \mathrm{dt}$, and muzzle voltage probes were used. However, FFT of the $E_{y}$ data showed only minor differences between the solid and arcing contact cases. This is because the sensor is far removed from the source of the emission. The FFT of the transient waveforms is quite rich in the high frequency spectrum. This is partly because the magnitudes are indeed small and because the signal-to-noise ratio at high frequencies is large. In order to illustrate the effect of the contact the spectra data can be fit to a straight line on a log-log plot. A plot of this fit for the muzzle voltage and the in-bore $\mathrm{dB} / \mathrm{dt}$ data is shown in Fig. 7. The muzzle voltage (recorded at $50 \mathrm{MHz}$ ) showed the strongest correlation of frequency content between solid and arcing contacts. The solid contact produces the most benign frequency spectra. Also, two contact transitions when current is roughly at peak produce the most severe frequency content. Although not shown, a single contact and two late-time contact transitions produced frequency content slightly better than the most severe. This ranking is as expected considering the power dissipation at the contact. The frequency content for the FFT of the dB/dt data shows larger frequency components than the $V_{m}$ data. Since the $\mathrm{dB} / \mathrm{dt}$ was recorded in bore and close to the field source the $\mathrm{dB} / \mathrm{dt}$ data are able to better resolve the frequency content. Note that the sensor has a linear transfer function for frequencies less that $9 \mathrm{MHz}$. In the time domain, contact transition does not alter the peak of the $\mathrm{dB} / \mathrm{dt}$ data. Rather, the amount of high frequency content is increased for transitioned armature contacts. The peak in-bore $\mathrm{dB} / \mathrm{dt}$ in this series of experiments is roughly $250 \mathrm{~T} / \mathrm{s}$. All FFTs, however, indicate that the low frequency magnitudes fall by roughly a factor of 100 at $100 \mathrm{kHz}$.

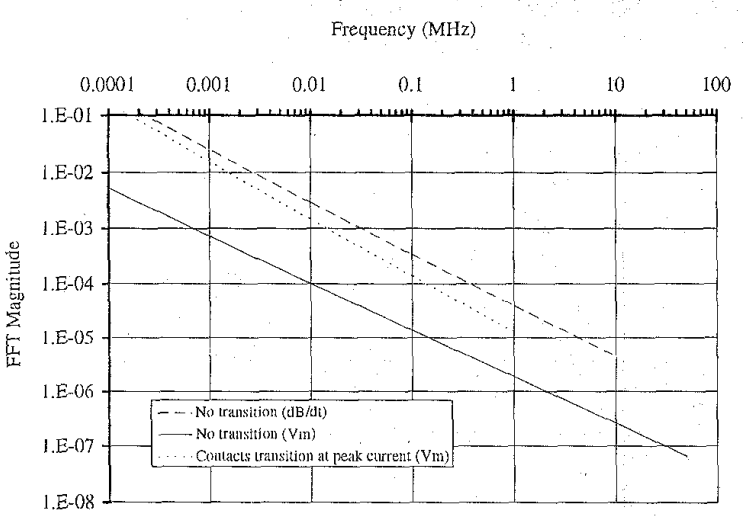

Fig. 7. Fit to frequency spectra for measured $\mathrm{dB} / \mathrm{dt}$ and $V_{m}$ data.

\section{Component Exposure Tests}

For these experiments six electronic packages were subjected to two types of environments: that produced by a solid contact and that produced by a transitioned contact. Their major functions were benchmarked in a simulator before exposure to the railgun in-bore environment. Additionally, because of the nature of field coupling to elec- 
tronics, a few of the electronics packages from each of the two contact groups were rotated $90^{\circ}$ from the main field component. After exposure, the electronic packages were subjected to acceptance tests to determine functionality.

The electronics package consisted of several major subassemblies and they are characterized by their significant electrical component. The three components are a microprocessor, an antenna, and a permanent magnet alternator. A summary of the in-bore exposure fields is listed in Table I. The smaller value listed for the E-field is inferred from the contribution by the armature while the larger value is inferred from the measured total E-field down range from the muzzle.

TABLE I

Summary of In-bore Exposure Fields

\begin{tabular}{|c|c|}
\hline$B_{z}(\mathrm{~T})$ & $E_{y}(\mathrm{kV} / \mathrm{m})$ \\
\hline 0.12 & $0.6-3.3$ \\
\hline
\end{tabular}

The microprocessor and antenna functions passed the acceptance tests. However, some degradation in the alternators output was observed. The degradation is characterized relative to the frequency and voltage output specifications for the alternator. It is suspected that the inbore B-field demagnetized the permanent magnets in the alternator. Hence, with less magnetization, there is less retarding force on the rotor of the alternator. Therefore, the rotor is able to spin faster and generate a higher frequency output. Also, the demagnetization causes the alternators magnetic field to be reduced, thereby producing a smaller output voltage. Despite the minor degradation, note that all six alternators functioned properly and each would perform its intended task at full performance. A summary of the results from the component tests and the type of exposure is listed in Table II.

TABLE II

Summary of Alternator Exposure Tests

\begin{tabular}{|c|c|l|}
\hline Contact & Orientation & Output Specifications \\
\hline Solid & $0^{\circ}$ & Frequency and voltage not met \\
\hline Solid & $90^{\circ}$ & Voltage not met \\
\hline Solid & $0^{\circ}$ & Frequency not met \\
\hline Arcing & $0^{\circ}$ & Frequency not met \\
\hline Arcing & $90^{\circ}$ & Voltage not met \\
\hline Arcing & $0^{\circ}$ & Met all specifications \\
\hline
\end{tabular}

\section{Summary and Conclusions}

The in-bore environment for a 50-mm square bore railgun was assessed. The armature was stationary. A quasistatic model for the in-bore B-field was developed from Biot and Savart's Law based on the launcher and armature current conducted in a thin sheet. Additionally, the azimuthal component of the magnetic field ahead of the armature was measured. The data are in good agreement with the model predictions.

The electric field was measured down range and exterior to the launcher. In prior experiments it was found that, in addition to the rail conductor system, external structures contributed significantly to the total environment. The model proved ineffective at predicting the E-field environment. Nevertheless, measured E-fields were used to scale the external environment to the in-bore armature location.

Data were recorded up to $50 \mathrm{MHz}$. The E-field and $\mathrm{dB} / \mathrm{dt}$ probes had linear transfer functions to $1 \mathrm{MHz}$ and $9 \mathrm{MHz}$, respectively. The voltage was measured with an active probe with a response up to $500 \mathrm{MHz}$. In all measurements, significant spectral content was less than $1 \mathrm{MHz}$. All low frequency magnitudes for $V_{m}, E_{y}$, and $B_{z}$, are reduced by at least 100 at $100 \mathrm{kHz}$. However, some frequency content exists above $1 \mathrm{MHz}$. Most notably was the narrow band peaks attributable to switching from the ignitrons in the capacitor banks. The most severe environment was produced by two armature contacts that transitioned near current peak.

Six electronics packages were subjected to the in-bore environment. Major subsystem components include a microprocessor, antenna, and an alternator. Microprocessor and antenna functions passed acceptance tests. The measured in-bore magnetic field of $0.12 \mathrm{~T}$ does not appear to have affected these components. The alternator was affected by the in-bore B-field environment. The permanent magnets used to provide excitation in the alternator were slightly demagnitized. The magnitude of the in-bore E-field was not quantified although its effect on these sensitive components appears to be nonexistent. The in-bore E-field values reported in this paper are not representative of the large caliber railgun environment.

Present and future railgun systems range from laboratory to mobile platforms, but the primary features are similar. The interior and exterior electric and magnetic field environment produced by the system is complicated and is dependent on the system configuration. The inbore issues were addressed relative to the functionality of an in bore electronics package for a stationary armature. Of greater concern is the case where the armature motion is not restrained. It is suspected that the time derivative of the electric and magnetic field quantities will be more pronounced, particularly at the time of projectile exit. Nonetheless, as was seen in the frequency spectra, the internal environment can becorne quite complicated due to coupling of external pulsed-power emissions.

\section{ACKNowledgments}

The authors would like to thank Mr. Ken Paxton for experimental support. Also, technical discussions with Dr. John Powell, ARL, were extremely useful in the design of the experiment. Mr. Lawrence Johnson, ARL, provided helpful discussions and guidance throughout this effort. The authors thank Mr. George McNally of the ARDEC Fuze Division (located at ARL) for graciously 
supplying the electronics packages and commitment to pre- and post- field exposure testing. Finally, funding was provided from the Electric Armaments Office, ARL.

\section{REFERENCES}

[1] Zielinski, A.E., Le, C.D., Bennett, J.A., and Kohlberg, I., "Railgun electric fields: theory and experiment," To be presented at the 9th IEEE Electromagntic Launcher Symposium, Edinburgh Scotland UK, May 1998.

[2] Heyse, M., Batteh, J.H., Scanlon, J.J., and Thornhill, L.D."Inbore measurements of the magnetic field in front of the armature and soft catch of electromagnetic launcher projectiles," Wright Laboratory Armament Directorate, Technical Report, WL-TR-967020, March 1996.

[3] Phillips, R.C. and Turner, E.B.,"Construction and calibration techniques of high frequency magnetic probes,", Review of Scientific Instruments, Vol 36, No 12, pp 1822-1825, December 1965.

[4] Roarke, R.J. and Young, W.C., Formulas for stress and strain, $5^{\text {th }}$ edition, McGraw-Hill Book Company,

[5] Marshall, R.A., "Current carrying capacity of solid armatures for railguns at zero speed," IEEE Tran. Magn., vol. 33, no. 1, pp 125-128, January 1997.

[6] Bauer, D.B. and Newman, D.C., "High performance railgun barrels for laboratory use," IEEE Tran. Magn.; vol. 29, no. 1 , pp 362-367, January 1993. 